Veritas Et Scientia

Vol. 7, N², 991-1001

Julio - Diciembre del 2018.

ISSN Edición Online: 2617-0639

https://doi.org/10.47796/ves.v7i2.76

\title{
PROPUESTA DE MODELO ASOCIATIVO PARA LA INTERNACIONALIZACIÓN DE LAS PEQUEÑAS Y MEDIANAS EMPRESAS AGROINDUSTRIALES DE ACEITUNA DE LA REGIÓN TACNA, 2016.
}

ASSOCIATIVE MODEL PROPOSAL FOR THE INTERNATIONALIZATION OF THE SMALL AND MEDIUM AGRIBUSINESS AGRICULTURAL COMPANIES OF THE TACNA REGION, 2016.

Luis Miguel Delgado Kuong ${ }^{1}$

Presentado: $14 / 07 / 2018$

Aceptado: $13 / 08 / 2018$

Publicado online:27/12/2018

\section{RESUMEN}

Objetivo: Formular una propuesta de modelo asociativo para la comercialización e internacionalización de las pequeñas y medianas empresas agroindustriales de aceituna de la Región Tacna en el 2016. Metodología: estudio no experimental, de relación y propositivo. Población: 50 empresarios y empresas resultados: el diagnóstico de la actividad agroindustrial de las pequeñas y medianas empresas agroindustriales de aceituna de la Región Tacna, es favorable (Matriz MEFE =3,23; Matriz MEFI =3,69). Conclusión: la propuesta de modelo asociativo para la comercialización e internacionalización de las pequeñas y medianas empresas agroindustriales de aceituna de la Región Tacna, es el Clúster Grupo Agro Olivo Tacna que tiene cinco ejes: Eje 1 Problemática identificada a través de un diagnóstico estratégico; Eje 2 Objetivos; Eje 3 Clúster olivícola e internacionalización; Eje 4. Factores de éxito y Eje 5. Desafíos, en razón a que generaría acometividad y mejora del posicionamiento de la aceituna en el mercado internacional.

Palabras clave: Asociatividad, internacionalización, empresas, agroindustria

\section{ABSTRACT}

Objective: focused on formulating a proposal for an associative model for the commercialization and internationalization of small and medium agroindustrial olive companies of the Tacna Region in 2016. Methodology: non-experimental, relationship and proactive study.

\footnotetext{
${ }^{1}$ Magíster en Management Internacional.
} 
Population: 50 entrepreneurs and companies' Main results: the diagnosis of the agroindustrial activity of the small and medium agroindustrial olive companies of the Tacna Region is favorable (MEFE Matrix = 3.23, MEFI Matrix = 3.69). Conclusion: the proposal of an associative model for the marketing and internationalization of small and medium agroindustrial olive companies of the Tacna Region, is the Group Agro Olive Tacna Cluster that has five axes: Axis 1 Problem identified through a strategic diagnosis; Axis 2 Objectives; Axis 3 Olivícola cluster and internationalization; Axis 4. Success factors and Axis 5. Challenges, because it would generate competitiveness and improve the positioning of the olive in the international market.

Keywords: Associativity, internationalization, companies, agribusiness.

\section{INTRODUCCIÓN}

El Ministerio de Producción del Perú (PRODUCE, 2016), afirmó que el segmento de mipymes constituye más del $99 \%$ del sector empresarial, el cual está preferentemente concentrado en el sector de servicios y comercio, ambos ligados con la demanda interna y muy poco articulados con los sectores de bienes transables. En Tacna, la Oficina Comercial de Exportación Regional, informó que se cuenta con 2500 Mypes principalmente de los sectores agro (olivos, orégano, páprika, cebolla, entre otros), pesca (abalones y mariscos en general), servicios (salud y software), textil y confecciones (ropa de algodón, prendas deportivas, mantas) y manufactura (muebles de madera y artesanías). Cabe subrayar, que la demanda de aceituna, se concentra en las aceitunas preparadas sin congelar (Colana Huarac, Juan Nicolás; Melgar Espíritu, Christian Abel; Moreno Torres, Rómulo Alberto; Muñoz Saldivar, 2017) (la que tiene la mayor preferencia), que representaron el $83 \%$ de la oferta exportable el 2013, las conservadas provisionalmente, las frescas o refrigeradas que registraron en el 2013 caídas del orden del 19\%, no así las aceitunas preparadas en vinagre o ácido acético que presentaron un crecimiento del orden del 73\%. En el 2015, los exportadores agrícolas en Tacna, alcanzaron un valor exportable de 182 millones de dólares, aun cuando esperaban lograr mayores cifras en exportación. La problemática de la internacionalización de la oferta agroindustrial de aceituna, se vincula con diferentes aristas: técnica, económica, social y legal, frente a lo cual se requiere de plantear alternativas de solución como por ejemplo analizar el potencial exportador de las empresas, diseñar un plan de iniciación y afianzamiento internacional, como también integrar asociaciones que les permita no solo fortalecer el gremio, sino también afrontar las barreras comerciales u obstáculos en orden a alcanzar la internacionalización, por tanto, es conveniente formular una Propuesta de Modelo de asociatividad que permita el desarrollo de las pymes agroindustriales de aceituna, como mecanismo para lograr mayor competitividad y capacidad de respuesta frente a la demanda internacional. Existe un vacío de conocimiento sobre el contexto integral de la realidad en que se desarrollan las empresas agroindustriales de aceituna y que limitan su internacionalización. Vásquez y Zapata (2015) en el Valle MotupeOlmos que el manejo de agro exportación de los productores de mango están en desarrollo, pero que existe una falta de financiamiento para los productores que no tiene acceso al crédito y podría ser un factor importante que está limitando su producción y exportación. Espinoza D. (2009) en Sao Paulo- Brasil, halló que la producción de aceitunas en el Perú va en 
aumento, debido al mayor volumen de aceitunas requerido. En Chiclayo, Soto (2014) refiere que existe falta de información, capacitación y asistencia técnica, entre los pequeños productores. Aybar, Huamaní, Olano, Sáenz. (2007) en llo se afirma que el proyecto de creación de valor para una nueva empresa modelo, incrementará el valor del patrimonio de los accionistas y sostenibilidad a los productores de aceitunas del sur peruano.

En Tacna, Quispe (2013) sostiene que el $42,7 \%$ de los integrantes de la cadena productiva de ceituna obtienen rendimientos entre $4001 \mathrm{~kg} / \mathrm{ha}$ y $5000 \mathrm{~kg} / \mathrm{ha}$; y que el 67,8\% reporta las transacciones de compra-venta en su propia parcela; solo el $37,8 \%$ de los integrantes de la cadena destinan su producción al mercado exterior. Latorre (2012 concluye que existe una influencia individual no significativa del insumo trabajo $(p>0,05)$, muy significativa del insumo capital $(p<0,05)$.

Quispe. (2013) en La Yarada, Región Tacna concluye que existe una relación significativa entre el nivel educativo de los productores, los proyectos ofrecidos, la asistencia técnica recibida, y el nivel de cumplimiento de los acuerdos.

La asociatividad empresarial para Latinoamérica y para las PyMES representa una ventaja importante como forma de aglomeración frente al reto de competitividad. Rosales (1997) define la asociatividad en PyMES, como un mecanismo de cooperación entre empresas pequeñas y medianas, en donde cada empresa participante, manteniendo su independencia jurídica y autonomía gerencial. Asimismo, la Cámara Comercio de Cartagena (s.f) considera que la asociatividad desarrolla mecanismos de acción conjunta y cooperación empresarial. Según Gomes y Cols. (2002) la cadena productiva se basa en el paradigma sistémico, y comprende los proveedores de insumos, las fincas y agroindustrias, los procesos productivos, las unidades de comercialización y los consumidores, los cuales en conjunto están vinculados por los flujos de capital, de información y materiales.

Las condiciones básicas para la asociatividad son la confianza, compromiso, definición de roles, términos de la membresía, liderazgo de gestión (Mathews, 2014), penalidades y sanciones y contrato integral. Según AFI (2010) los beneficios de la internacionalización de las empresas presentan un aumento volumen de facturación como en tamaño de plantilla, son más competitivas, generan más y mejor ocupación y resisten mejor a ciclos adversos (ICEX \& AFI, 2010).

\section{METOdOLOGÍA}

Investigación de enfoque mixto, propositivo, explicativo no experimental. La población es las pequeñas y medianas empresas agroindustriales de aceituna $(n=50)$ registradas en la Cámara de Comercio, Industria y Producción de Tacna. Para la investigación, se aplicó un cuestionario validado por juicio de expertos. Se estableció un código maestro. Para la interpretación de los hallazgos, se analizó la tendencia, el comportamiento, los hechos según la cronología y el contexto coyuntural del estudio, a fin de pronosticar y encontrar, mediante estimaciones estadísticas las relaciones entre estos datos en grados de probabilidad, incertidumbre o certeza.

\section{RESULTADOS}

Según el Ministerio de Agricultura y Riego (2016) del Perú, en el periodo de enero a mayo 2016, se reportó una producción de 14,2 mil toneladas ( $131 \%$ de crecimiento), a diferencia del 
2015 en la que la producción alcanzó una cifra de 18,2 mil toneladas, siendo la producción mayor en la región sur, especialmente Tacna y Arequipa.

Solo en mayo del 2016, la diferencia en términos de incremento fue de 8 mil toneladas, ya que el 2015 fue de 10,4 y el 2016 se elevó a 18,4 mil toneladas (77\% más), siendo Arequipa la que mostró el crecimiento más agresivo respecto a los demás departamentos del Perú. (Figura 1).

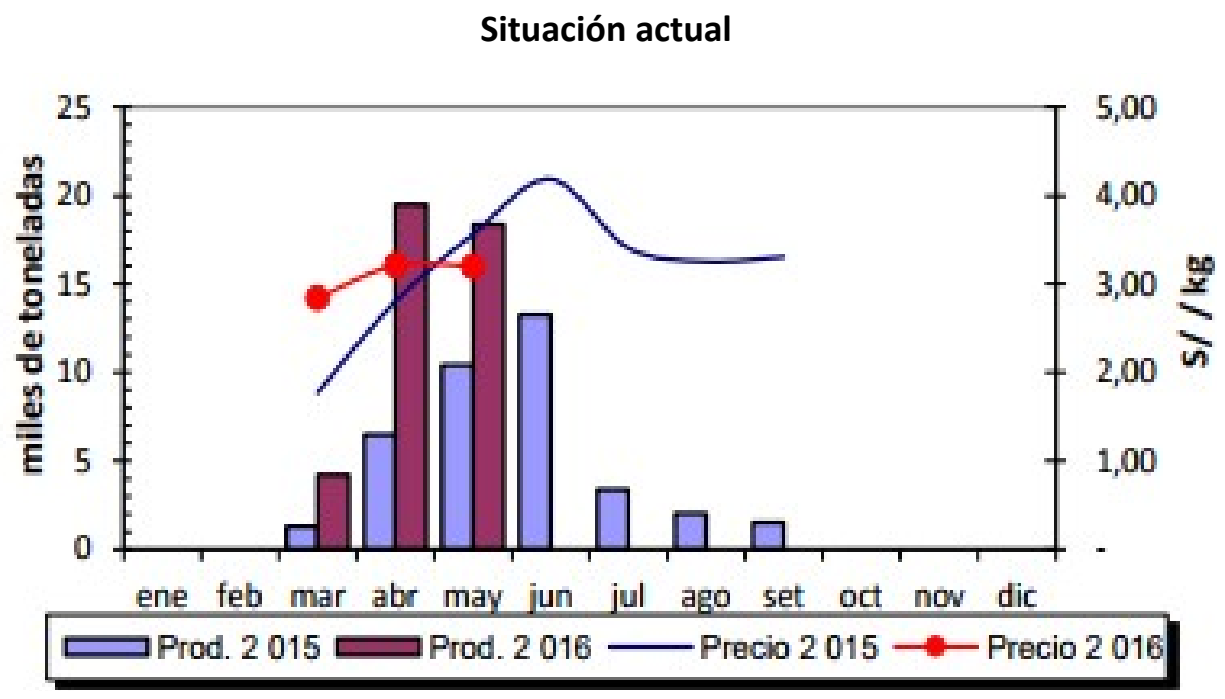

Figura 1. Producción de aceituna. Fuente: Ministerio de Agricultura y Riego

La variación porcentual del valor bruto de la producción de aceituna (millones de soles) en el 2016-2015 fue de 130,7 para la aceituna en el Perú, la cifra más alta alcanzada en los últimos tres años, a diferencia del 2012 que alcanzó una cifra de 140,6. La variación porcentual del precio recibido por la aceituna (soles/tonelada) para el 2016-2015 fue de 0,2, a diferencia del periodo 2015-2014 que presentó una variación porcentual de 123,6.

En el ranking de Competitividad el Perú en general se ubica en el puesto 58, también el riesgo país a fines del 2017 se ubicó en 1,4 y la inflación ha decrecido en el 2017 a comparación del 2016, lo cual, representa un panorama propicio para la inversión para el desarrollo del sector olivicultor. (Tabla 01).

Según las fuerzas tecnológicas y científicas (Tabla 02), en general, el Perú se encuentra en una posición desventajosa frente a otros países, lo que al parecer según Villarán (2011) tendría una explicación entre los factores que se consideran los más problemáticos para realizar negocios como burocracia gubernamental ineficientes, corrupción, regulaciones laborales restrictivitas, entre otras. De otro lado, existe el Plan Nacional de CTI 2006 - 2020 para impulsar la ciencia y la tecnología, cuyo propósito es promover el desarrollo y la transferencia de la ciencia, promover la mejora del capital humano en términos cualitativos y cuantitativos, fortalecer la articulación de los diversos sectores para la institucionalización de la tecnología e innovación, alineado con el Sistema Nacional de Planeamiento estratégico del Perú. (Tabla 03)

La matriz del sector olivícola en la provincia de Tacna, cuenta con 15 factores de éxito (8 oportunidades y 7 amenazas). El resultado ponderado total para el sector olivícola, alcanza un valor de 3,23, lo que evidencia que el sector olivícola requiere de un esfuerzo que permita vulnerar las amenazas, teniendo en cuenta que las oportunidades se presentan propicias actualmente. 
Tabla 01

Fuerzas económicas y financieras (PESTE)

\begin{tabular}{l|l}
\hline & \multicolumn{1}{c}{ Descripción } \\
\hline Fuerzas & $-\begin{array}{l}\text { Según el Banco Central de Reserva, la inflación anual en el Perú ha } \\
\text { disminuido desde 3,2\% en diciembre 2016 a 1,4 a diciembre de } \\
\text { económico } \\
\text {-financieras }\end{array}$ \\
& $\begin{array}{l}\text { agrícolas, el déficit de recursos hídricos a fines de 2016 y el } \\
\text { Fenómeno del Niño Costero a inicios del 2017. (El Comercio, 2017) }\end{array}$ \\
& $-\begin{array}{l}\text { El riesgo país de Perú al 12.12. 2017 cerró en 1,12 según el banco de } \\
\text { inversión JP Morgan (Diario Gestión, 2017) }\end{array}$ \\
& $-\begin{array}{l}\text { FOB para la aceituna según el MINCETUR (2016) es de \$1,74 } \\
\text { superior en un 2,9\% respecto del 2015 }\end{array}$ \\
\hline
\end{tabular}

Tabla 02

Fuerzas tecnológicas y científicas (PESTE)

\begin{tabular}{|c|c|}
\hline & Descripción \\
\hline $\begin{array}{l}\text { Fuerzas } \\
\text { tecnológicas y } \\
\text { científicas }\end{array}$ & $\begin{array}{l}\text { - } \quad \text { Perú se ubica en el puesto } 86 \text { de tecnología, entre } 148 \text { países } \\
\text { - } \quad \text { Perú tiene el puesto } 122 \text { en innovación, entre } 148 \text { países } \\
\text { - } \quad \text { Plan Nacional de Ciencia Tecnología e Innovación (CTI) 2006-20 }\end{array}$ \\
\hline
\end{tabular}

Tabla 03

Fuerzas ecológicas y ambientales (E) (PESTE)

\begin{tabular}{|c|c|}
\hline & Descripción \\
\hline $\begin{array}{l}\text { Fuerzas } \\
\text { ecológicas y } \\
\text { ambientales }\end{array}$ & $\begin{array}{ll}- & \text { Sistema Nacional de gestión Ambiental (MINAM, 2016) } \\
\text { - } & \text { Ley del Sistema Nacional de Evaluación del Impacto Ambiental № } \\
& 27446 . \\
- & \text { Protocolo de Kioto } \\
- & \text { Plan Nacional de Acción Ambiental (PLANNA) } \\
- & \text { Autoridad Nacional del Agua (ANA) del Ministerio de Agricultura y } \\
& \text { Riego }\end{array}$ \\
\hline
\end{tabular}

Fuente: elaboración propia

En la matriz DAFO para el sector olivícola de la provincia de Tacna, se aprecian 19 factores determinantes de éxito (10 fortalezas y 9 debilidades). El valor global denota que el sector olivícola tiene muchas posibilidades para expandirse, fortalecerse y crecer, lo que amerita definir actividades estratégicas para mejorar su posicionamiento e integración para fines de posicionarse en el mercado internacional. Según la valoración de la Matriz de Rumelt, del análisis realizado, se aprecia que la estrategia Promover encadenamiento productivo con Arica para generar volumen A7 F1, no califica en el criterio ventaja, lo que supone que no está 
alineada en los cuatro filtros, por tanto, no se aprueba. En todos los demás casos, se alinean completamente.

Tabla 04

Empresarios del sector olivícola de la producción de la aceituna en Tacna, 2017

\begin{tabular}{|c|c|c|}
\hline & №=50 & $\%=100$ \\
\hline \multicolumn{3}{|l|}{$\begin{array}{l}\text { Supervisa los procesos y actividades de los } \\
\text { trabajadores para asegurar eficiencia y calidad del } \\
\text { proceso y producto }\end{array}$} \\
\hline Nunca & 7 & 14,0 \\
\hline A veces & 21 & 42,0 \\
\hline Siempre & 22 & 44,0 \\
\hline \multicolumn{3}{|l|}{$\begin{array}{l}\text { Aplica estrategias para seleccionar recursos } \\
\text { humanos calificados }\end{array}$} \\
\hline Nunca & 4 & 8,0 \\
\hline A veces & 24 & 48,0 \\
\hline Siempre & 22 & 44,0 \\
\hline \multirow{2}{*}{\multicolumn{3}{|c|}{$\begin{array}{l}\text { Coordina la administración de los recursos } \\
\text { financieros de la empresa para alcanzar los } \\
\text { objetivos trazados }\end{array}$}} \\
\hline & & \\
\hline A veces & 27 & 54,0 \\
\hline Casi siempre & 4 & 8,0 \\
\hline Siempre & 19 & 38,0 \\
\hline \multicolumn{3}{|l|}{$\begin{array}{l}\text { Coordina la administración de los recursos } \\
\text { materiales de su empresa }\end{array}$} \\
\hline A veces & 27 & 54,0 \\
\hline Siempre & 23 & 46,0 \\
\hline \multicolumn{3}{|l|}{$\begin{array}{l}\text { Coordina la administración de los recursos } \\
\text { humanos de su empresa }\end{array}$} \\
\hline A veces & 35 & 70,0 \\
\hline Siempre & 15 & 30,0 \\
\hline \multicolumn{3}{|l|}{$\begin{array}{l}\text { Coordina la administración de los recursos } \\
\text { tecnológicos de su empresa }\end{array}$} \\
\hline A veces & 30 & 60,0 \\
\hline Siempre & 20 & 40,0 \\
\hline
\end{tabular}

Se evidenció que en su mayoría los empresarios encuestados, supervisan los procesos y actividades que se realizan en su empresa para garantizar la eficiencia y la calidad no solo de los procesos sino también de los productos (44\%), aunque una proporción considerable (42\%) manifiesta que a veces supervisa. De otro lado, para el reclutamiento de recursos humanos, en su mayoría los empresarios a veces aplican estrategias para seleccionar recursos humanos calificados (48\%). También, cuando se trata de coordinar la administración de los recursos financieros destinados al logro de objetivos estratégicos, un $54 \%$ admite que a veces coordina $y$ un $38 \%$ manifiesta que siempre lo hace en forma coordinada. En el mismo sentido, un $54 \%$ coordina la administración de recursos materiales y en cuanto a la gestión de recursos 
humanos un $70 \%$ asiente que a veces coordina su administración. La misma tendencia, se observa cuando se le consulta sobre si coordina la administración de los recursos tecnológicos para la empresa, ya que un $60 \%$ reconoce que a veces realiza tal coordinación, la diferencia $40 \%$ dice que siempre lo realiza en forma coordinada (Tabla 04).

En cuanto a la percepción de los empresarios del sector agroindustrial olivícola, se halló que mayormente aplican siempre estrategias de motivación para una mejorar producción (68\%), promueven el trabajo en equipo y la comunicación (66\%), aseguran condiciones de bienestar y seguridad (66\%). De otro lado, más de la mitad, admite que a veces meritúa que sus decisiones que asume como director o empresario o dueño de la agroindustria, le permiten realmente alcanzar los objetivos que se ha propuesto.

Asimismo, los empresarios manifiestan que siempre consideran estándares de desempeño para cada actividad y puesto de trabajo (66\%), aunque prácticamente una cuarta parte (26\%) considera que a veces. Respecto a si evalúa los resultados con los indicadores planificados, las dos terceras partes (66\%) manifiesta que siempre lo hace. En cuanto a si tienen establecidas medidas correctivas para las actividades y resultados que no resulten satisfactorios, un $60 \%$ respondió que siempre, mientras que un $20 \%$ en cada caso responde que nunca y a veces. De otro lado, la mayoría de los empresarios manifiesta que siempre (80\%) planifica la obtención y uso de los recursos financieros para su empresa, la diferencia responde que a veces (20\%).

La mayoría admite que los recursos financieros que utiliza para afrontar la inversión y gastos los obtiene principalmente de entidades financieras crediticias (54\%) (v.g. cooperativas, bancos). Asimismo, casi las dos terceras partes (60\%) de los empresarios perciben que siempre consideran que el manejo financiero es adecuado para obtener la productividad y rentabilidad que esperan, lo cual sugiere que los resultados de producción fueron favorables. Un punto interesante, es que cuando se les pregunta si presupuestan para invertir en nuevas instalaciones, equipos, materia prima, insumos y productos terminados, más de la mitad (58\%) responde que siempre lo hacen, prácticamente un tercio indican que a veces (34\%). Igualmente, la planificación de adquisición y aplicación de recursos tecnológicos es minoritaria, ya que solo el $46 \%$ manifiesta que siempre es parte de su planificación, sin embargo, un $40 \%$ dice que a veces y un $14 \%$ lo que sugeriría que es posible que aún para muchos la inversión en tecnología o maquinaria de última generación representa un gasto más que una inversión. En cuanto a la gestión de los recursos humanos, un $54 \%$ revela que siempre incentiva a su personal a capacitarse, un tercio (32\%) dice que a veces y un $14 \%$ expresa que nunca realiza tal motivación (Tabla 05).

Cuando se exploró sobre la intencionalidad o interés por asociarse, casi las dos terceras partes (62\%) manifiesta un interés claro, sin embargo, solo el $18 \%$ tiene una propuesta de plan exportador, lo que evidencia quizá desconocimiento o una intencionalidad lejana o improbable. De otro lado, solo un $48 \%$ tiene conocimiento sobre requisitos estándares que deben cumplir la materia prima, envase, empaques y proveedores. Más de la mitad de los empresarios, expresaron poseer un sistema de gestión de calidad (52\%), un $18 \%$ una certificación de calidad y un $20 \%$ dice que siempre capacita a su personal sobre la calidad en producción y servicios.

Tabla 05

Procedencia de financiamiento de la producción de la aceituna

$\mathrm{N}=50$

Veritas et Scientia Vol. 7 (2) Julio - diciembre 2018. 


\begin{tabular}{lcc}
\hline $\begin{array}{l}\text { Los recursos financieros para las actividades de la } \\
\text { empresa provienen: }\end{array}$ & \\
Recursos propios (ahorros, utilidades ) & 13 & 26,0 \\
Recursos ajenos (préstamos, créditos bancarios) & 27 & 54,0 \\
Ambos & 10 & 20,0 \\
\hline Considera que el manejo financiero es adecuado & & \\
para obtener productividad y rentabilidad & & 40,0 \\
A veces & 20 & 60,0 \\
Siempre & 30 & \\
\hline Presupuesta los recursos para nuevas & & \\
instalaciones, equipos, materias primas, insumos & & 8,0 \\
y productos terminados & & 34,0 \\
Nunca & 4 & 58,0 \\
A veces & 17 & \\
Siempre & 29 & 14,0 \\
\hline Planifica la adquisición y aplicación de recursos & & 40,0 \\
tecnológicos & & \\
Nunca & 7 & \\
A veces & 20 & \\
Siempre & 23 & 14,0 \\
\hline Incentiva a su personal a capacitarse y & & 32,0 \\
actualizarse & & 54,0 \\
Nunca & 7 & \\
A veces & 16 & \\
\hline
\end{tabular}

Se halló que el $88 \%$ de las empresas agroindustriales olivícolas, no tienen un área de investigación y desarrollo, en su gran mayoría tampoco aplican vigilancia tecnológica (94\%), no verifican los requisitos legales de producción (80\%). Un $58 \%$ si tiene una política como empresa para cuidar responsablemente el medio ambiente, ya que el $34 \%$ manifiesta que siempre cuida de minimizar el impacto ambiental por desechos de producción y otras acciones derivadas de las actividades que realizan como consecuencia de sus acciones agroindustriales.

\section{DISCUSIÓN}

Respecto a los hallazgos empíricos, de la información recogida de los empresarios de agroindustrias del olivo, se tiene que en cuanto a la gestión y específicamente a la formulación de objetivos y estrategias, la mayoría lo realiza en conjunto con su grupo familiar, basados en su experticia, pero presumiblemente en muchos casos alejados de las herramientas de gestión moderna que les permita optimizar la gestión de los recursos humanos, financieros, operativos, estratégicos e incluso personales, lo que sin duda, en muchos casos limita su consolidación y crecimiento (Avolio, Mesones, Roca, s.f.). Se debe tener en cuenta que la definición de objetivos y estrategias es quizá uno de los factores más relevantes para el éxito organizacional. Los resultados en cuanto a la gestión motivacional, de promoción de trabajo en equipo y de dotar de condiciones de seguridad y de bienestar al personal, son favorables, ya que en su mayoría (más de la mitad) los empresarios manifiestan que siempre se preocupan por favorecer este tipo de gestión, lo cual, explicaría que a pesar de que la mayoría en muchos aspectos presenta una gestión basada en la experiencia más que técnica o profesional, los 
resultados son positivos. En tal sentido, si se capacita al personal que lidera la gestión de las Mypes con herramientas de gestión estratégica, es posible que se optimice la tarea que actualmente realizan, obteniendo aún mejores resultados.

Teniendo en cuenta que la mayoría (66\%) respondió que siempre tiene establecidos estándares de desempeño para determinada actividad o puesto de trabajo, lo cual es positivo, es importante indicar que se requiere estandarizar los parámetros para evaluar el desempeño del trabajador según el perfil del puesto, para una medición objetiva. Asimismo, las ventajas de una evaluación del desempeño y un feedback continuo, minimizan los errores y permite aplicar medidas correctivas tendientes a maximizar, en orden a alinear las habilidades del personal hacia el cumplimiento de la misión. En lo referente a la contrastación de los resultados con los indicadores planificados, es relevante manifestar que la evaluación de los indicadores de producción, rendimiento de maquinaria y tecnología, logística, productividad según recurso humano, impacto ambiental (Estupiñan, 2005), entre otros, contribuye con la mejora continua de la empresa, con mejores resultados para diferenciarse de la competencia y por ende alcanzar una mayor competitividad. También se destacan los hallazgos, de un $60 \%$ admite que a priori siempre tiene establecidas medidas correctivas para las actividades y resultados que no resulten satisfactorios; lo cual es favorable, ya que los sistemas de gestión de calidad en general, consideran una herramienta de calidad el "manejo de las acciones correctivas" (Zuta y Wiese, 2013.) En cuanto a la obtención y uso de los recursos financieros en las empresas agroindustriales olivícola, se alude a que "uno de los talones de Aquiles de las PyMES se encuentra en el proceso de planificación financiera" (Ortiz, 2017, párr.1), ya que nos prepara para hacer frente a los retos, desafíos y riesgos del futuro, ya que nos delinea una ruta y un norte, hacia el cual nos dirigimos y especialmente, como deberían usarse los recursos financieros dentro del contexto de esta panorámica.

También se encontró que la mayoría de los empresarios financia sus actividades e inversión empresarial de recursos provenientes de empresas crediticias, lo que nos permite presumir que, a pesar de las exigencias de las financieras, los empresarios son sujeto de crédito. Es importante merituar, que se considera un factor de accesibilidad a créditos privados la asimetría de la información cuando se trata de tramitar por un lado y aprobar un financiamiento para una empresa pequeña un crédito, ya que muchas veces los que suministran el financiamiento no poseen una información completa de la empresa y sus características, de tal forma, que generalmente se presume que existe un alto riesgo de impago por parte de ésta, lo que limita en gran medida la posibilidad de ser sujeto de crédito (Nieto, 2017). Sin embargo, según la información de los empresarios más de la mitad accede a este tipo de financiamiento, lo que denotaría confianza y oportunidad que favorece el crecimiento de las MyPES.

Otro aspecto interesante, que se revela en los hallazgos es que la mayoría de los empresarios, no utiliza las redes sociales para fines de mercadeo, solo el $12 \%$ lo usa siempre, lo que a nuestro criterio representa una debilidad ya que actualmente, la tendencia del uso del internet como herramienta para la búsqueda de servicios y productos es abrumadora. Estos resultados coinciden con los hallazgos de Carreño, Orellana, Pesantez, Loor (2015) quienes hallaron que las pymes en Ecuador no utilizan adecuadamente las herramientas que el marketing y las TICs les proporcionan para mejorar su desarrollo empresarial. Asimismo, se ha minimizado la elaboración de planes de mercadeo, solo el $6 \%$ lo realiza siempre, la diferencia lo hace a veces o nunca, es decir, solo una minoría tiene la posibilidad de definir los mercados que va a cubrir, los segmentos a los que se dirige principalmente el producto, y adicionalmente sin que sea menos importante, ayudar a generar ventajas competitivas y una posición distintiva y diferenciadora de la empresa frente a la competencia (Hoyos, 2010), de tal forma que la marca 
se consolide y se torne poderosa. Del mismo modo, se ha soslayado las estrategias de penetración y posicionamiento en otros mercados, ya que solo el $8 \%$ en cada caso lo realiza, lo cual restringe su crecimiento, a pesar de que deberá tenerse en cuenta que la piedra angular es la suficiencia de la empresa para diferenciarse y desarrollar la marca. Un resultado también importante, fue que solo el $8 \%$ se preocupa por el volumen de ventas, aspecto que para fines de exportación es necesario considerar que el requerimiento de volumen de aceituna, excede muchas veces la capacidad de oferta de un empresario, lo que implicaría la necesidad de asociarse. Más de la mitad de los empresarios (52\%) no tiene suficientes conocimientos sobre los requisitos estandarizados que deben cumplir la materia prima, los envases, empaques y sobre la gestión de proveedores, lo que constituye una debilidad, no solo para obtener un producto de calidad, sino para tener una mayor posibilidad de internacionalizar la aceituna y productos derivados. Se encontró también que solo el $40 \%$ realiza una selección previa de proveedores, lo que resulta preocupante, ya que éstos son elementos vitales para que fluya el proceso comercial, más aun si se considera que la calidad de las entradas determina la calidad de las salidas (Farfan, 2014), considerándose con razón aliados estratégicos. La preocupación actual de toda empresa es sin lugar a dudas anticiparse para prevenir las posibles amenazas o para aprovechar las oportunidades y eludir una "gestión de carácter exclusivamente reactiva" (Hidalgo, 1998, p.1), en este sentido, el encontrar como resultado que solo el $6 \%$ de las empresas de olivo en Tacna, aplique vigilancia tecnológica, disminuye su posibilidad de aprovechar la posibilidad de identificar las innovaciones en lo concerniente al producto, servicio u objeto materia de interés, su valor comercial actual y las posibles amenazas de carácter tecnológico que podrían provenir de otras empresas del mismo rubro. De los hallazgos también se evidencia, que la modalidad de asociatividad que se ajusta en mayor medida a la información que arroja el análisis estratégico, es un clúster olivícola, basado en intereses comunes, que genere una confianza entre los empresarios, para beneficiar a toda la cadena de comercialización del olivo en Tacna. Sin embargo, se presentan limitantes como las deficiencias de cultura empresarial, relacionadas con la comercialización, estándares de calidad, conocimientos metodológicos para lograr la internacionalización de sus productos.

La asociatividad tipo clúster es la postura estratégica más apropiada para el sector olivícola para fines de comercialización e internacionalización, ya que la intersección del total de la matriz MEFE y MEFI se ubica en el cuadrante I, lo cual determina pertinente desarrollar acciones para crecer y construir, asumir estrategias intensivas y de integración como la asociatividad tipo clúster para el secto

\section{REFERENCIAS BIBLIOGRÁFICAS}

Aybar G., Huamaní N., Olano R., Sáenz A. (2007). Estrategia exportadora para el sector de aceitunas del sur peruano al mercado estadounidense: aplicación del modelo Exporting Trading Companies (ETC) en la empresa Aceitunas de llo S.A.

Avolio B., Mesones, A., \& Roca, E. (2011). Micro y Pequeñas Empresas en el Perú (MYPES). Centrum católica, 1(1), 70-80

Cámara Comercio de Cartagena (s.f) Fortalecimiento empresarial. Asociatividad empresarial.

Carreño, Orellana, D. Pesantez, R. Loor, A. (2015). Las Herramientas del Marketing y las TIC's: su Uso en las Pymes para el Desarrollo Empresarial, 7, 73-87.

Colana, J.; Melgar, C.; Moreno, R.; Muñoz, J. E. (2017). Planeamiento Estratégico de la Aceituna en el Perú 
Espinoza D. (2009). Factores de crecimiento de las exportaciones de aceitunas de mesa sevillana (Olea europea) a Sao Paulo Brasil (2004-2008) (Tesis de titulación) Universidad San Martín de Porras, Lima.

Estupiñan, A. (2005). Diseño de indicadores de producción en la industria de alimentos de Barranquilla y Cartagena. Avances En Ingeniería Y Tecnología, 8-9.

Farfán, A. (2014). La Gestión de Relación con los Proveedores en la Cadena de Abastecimiento Supplier Relationship Management in the Supply Chain. Sinergia E Innovación Consultado de Sinerg. Innov, 2(22), 118-122.

Gestión. (2014). Oficina Comercial de Exportación Regional de Tacna beneficiará a 2,500 MyPES. 28.02.2014.

Gomes de Castro, A., et. al. (2002). Cadena productiva: marco conceptual para apoyar la prospección tecnológica

Hidalgo, A. (1998). Vigilancia tecnológica: su importancia estratégica en la empresa, (March).

Hoyos, R. (2010). El papel del marketing en las Empresas: misión, objetivos y funciones. Papeles de Administración, (6), 12-20.

ICEX \&AFI (2010). Internacionalización, empleo y modernización de la economía española [En línea] [Fecha de consulta: 9 de mayo de 2017] Recuperado de

Mathews J. (2014). Asociatividad empresarial, Universidad del Pacífico, Lima.

Nieto, M. M. (2017). Factores de crecimiento y rentabilidad de las MyPES del sector industrial de Villa El Salvador - Lima, 2016. Universidad Inca Garcilazo de la Vega. [Fecha de consulta: 3 de mayo de 2017]

Ortiz, J. (2017). PROCESO DE PLANIFICACIÓN FINANCIERA DE UNA PYME. Retrieved March 18, 2018, [Fecha de consulta: 18 de marzo de 2018]

PRODUCE. (2016). Anuario Estadístico Industrial, Mipyme y Comercio Interno 2015. Ministerio de La Producción Del Perú, 138.

Quispe R. (2013). Evaluación de la cadena productiva del Olivo (Olea europea) en la Irrigación La Yarada, Región Tacna. (Tesis de Licenciatura). Universidad Nacional Jorge Basadre Grohmann, Tacna.

Rosales R. (1997). La asociatividad como estrategia de fortalecimiento de las PYMES. Universidad de Texas, Texas.

Zuta, I., \& Wiese, A. M. (2013). Una Mype Con Calidad. 